Check for updates

Cite this: RSC Adv., 2017, 7, 43512

Received 18th July 2017

Accepted 18th August 2017

DOI: $10.1039 / \mathrm{c} 7 \mathrm{ra07908g}$

rsc.li/rsc-advances

\title{
A cellulose-based hybrid 2D material aerogel for
a flexible all-solid-state supercapacitor with high \\ A cellulose-based hybrid 2D material aerogel for
a flexible all-solid-state supercapacitor with high specific capacitance $\uparrow$
}

\author{
Yanyan Lv, ${ }^{a}$ Lei Li, ${ }^{a}$ Yi Zhou, (D) a Miao Yu, ${ }^{a}$ Jianquan Wang, ${ }^{a}$ Jianxin Liu, ${ }^{a}$ Jiagui Zhou, ${ }^{b}$ \\ Zongqing Fan $^{\mathrm{b}}$ and Ziqiang Shao (D) *a
}

\begin{abstract}
As one of the energy storage devices, all-solid-state flexible supercapacitors have attracted significant attention because of their high power density, low cost, high safety, low environmental impact, and long cycle life. In this study, a new type of all-solid-state flexible supercapacitor that uses cellulose nanofibers (CNFs)/molybdenum disulfide $\left(\mathrm{MoS}_{2}\right) /$ reduced graphene oxide (RGO) hybrid aerogel film as an electrode material and charge collector and $\mathrm{H}_{2} \mathrm{SO}_{4}$ /polyvinyl alcohol (PVA) gel as an electrolyte and separator has been demonstrated. These aerogels are prepared by supercritical $\mathrm{CO}_{2}$ drying, which use $\mathrm{CNFs}$ as an effective, environmentally friendly, and steady dispersant of $\mathrm{MOS}_{2}$ and RGO. Owing to the porous structure of the electrodes and the remarkable electrolyte absorption properties, the supercapacitors exhibit excellent electrochemical properties. The specific capacitance calculated from the cyclic voltammogram curves at a scan rate of $2 \mathrm{mV} \mathrm{s}^{-1}$ is about $916.42 \mathrm{~F} \mathrm{~g}^{-1}$. The capacity retention is more than $98 \%$ after 5000 charge-discharge cycles at a current density of $0.5 \mathrm{~mA} \mathrm{~cm}^{-2}$. Additionally, the areal capacitance, areal power density, and energy density of the supercapacitors are about $458.2 \mathrm{mF} \mathrm{cm}^{-2}$, $8.56 \mathrm{~mW} \mathrm{~cm}{ }^{-2}\left(4.3 \mathrm{~kW} \mathrm{~kg}^{-1}\right)$, and $45.7 \mu \mathrm{W} \mathrm{h} \mathrm{cm}{ }^{-2}\left(22.8 \mathrm{~W} \mathrm{~h} \mathrm{~kg}^{-1}\right)$, respectively. Owing to its low cost and environmental friendliness, $\mathrm{CNFs} / \mathrm{MoS}_{2} / \mathrm{RGO}$ nanohybrid aerogel has great application potential as an electrode material of all-solid-state flexible supercapacitors.
\end{abstract}

\section{Introduction}

Along with economic development and technological progress, the demand for high-performance energy storage devices is increasing. All-solid-state flexible supercapacitors possess the following advantages: low cost, lightweightness, high power and energy density, excellent durability, high flexibility, and environmental friendliness; moreover, they are considered to be promising for portable and wearable electronics such as roll-up displays and electric paper. ${ }^{1-6}$ Their performance mainly depends on the physicochemical processes, such as ion transfer, charge transfer, and electrode wetting by the electrolyte, at electrode-electrolyte interfaces; therefore, electrode materials with excellent electrochemical properties and appropriate microstructure are crucial for high performance all-solid-state flexible supercapacitors. ${ }^{7-9}$

\footnotetext{
${ }^{a}$ Beijing Engineering Research Centre of Cellulose and Its Derivatives, School of Materials Science and Engineering, Beijing Institute of Technology, Beijing 100081, P. R. China. E-mail: shaoziqiang@263.net

${ }^{b}$ Hubei Jinhanjiang Refined Cotton Co, Hubei 431900, P. R. China

$\dagger$ Electronic supplementary information (ESI) available: The preparation of CNFs Suspension, $\mathrm{MoS}_{2}$ powder and GO suspension; the calculation of the electrochemical parameters; photograph of nanohybrid aerogel, nanohybrid aerogel film and nanohybrid aerogel all-solid-state flexible supercapacitor; the comparison of electrode capacitance. See DOI: 10.1039/c7ra07908g
}

Two-dimensional (2D) materials, such as MXene, molybdenum disulfide $\left(\mathrm{MoS}_{2}\right)$, and graphene, are outstanding candidates for applications in flexible energy storage devices because of their multifunctional electronic structure and relatively high specific surface area. Graphene-based porous carbon materials have been studied in the past for applications in flexible electrochemical energy storage devices because of their many excellent properties such as high specific surface area $\left(2675 \mathrm{~m}^{2} \mathrm{~g}^{-1}\right)$, strong mechanical behaviour, and rapid thermal and electrical conductivity; however, their capacity is relatively low. ${ }^{10-15} \mathrm{MoS}_{2}$ sheets, a kind of layered 2D materials obtained from transition metal dichalcogenides (TMDs), which have similarities with graphene, have been studied to a lesser extent; however, they have attracted increasing interest in recent years for integration into energy storage devices because they can provide a high specific surface area for charge storage of an electric double layer. ${ }^{16,17}$ But their applications alone in supercapacitors are still very limited because of their low specific capacitance, which is probably related to the low conductivity of the $\mathrm{MoS}_{2}$ molybdenite phase. ${ }^{18}$ In this regard, with the obvious advantages and disadvantages of these $2 \mathrm{D}$ materials, the combination of both materials can provide innovative hybrid materials, and they can be applied as promising electrochemical supercapacitor devices. However, owing to the unavoidable re-stacking, the ion diffusion of the electrolyte 


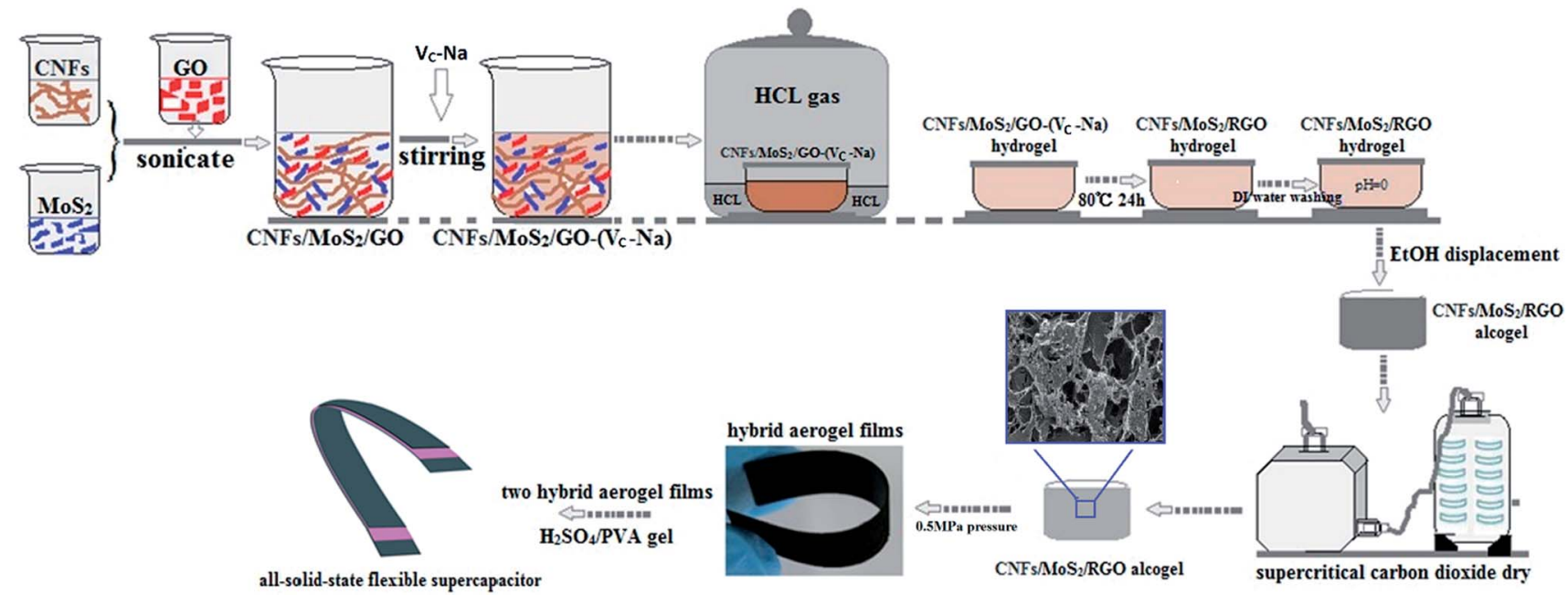

Fig. 1 Schematic of the preparation process of the CNFs/MoS $/$ RGO nanohybrid aerogel electrode films and the fabrication of the flexible allsolid-state supercapacitors.

becomes hard or even impossible in a tightly packed structure of reduced graphene oxide (RGO) nanosheets; thus, the specific surface area of RGO-based materials is apparently lower than the theoretical value. As a result, the performance of graphene-based supercapacitors is significantly degenerated. ${ }^{8,19-28}$ In addition, $\mathrm{MoS}_{2}$ is easy to reunite because of its 2D structure, which is analogous to that of graphene. ${ }^{29}$ Consequently, search for an advanced dispersant to effectively prevent graphene and $\mathrm{MoS}_{2}$ aggregation, improvement of the electrolyte wettability, and reduction of the ion diffusion distance are very important in improving the properties of $\mathrm{RGO} / \mathrm{MoS}_{2}$-based supercapacitors. ${ }^{7,30}$ As is known, cellulose is one of the most abundant, renewable resources on earth and possesses outstanding properties such as biocompatibility, environmental benignity, and desired chemical stability. With the development of nano-technology, it is gradually extending to nanochemicals, nano-processing technology, nano-composite materials, and other high value-added areas. ${ }^{31}$ As a type of cellulose, one-dimensional cellulose nanofibers (CNFs) have outstanding mechanical properties, high aspect ratios, prominent flexibility, and superior hydrophilicity. ${ }^{31,32}$ Additionally, CNFs have some other remarkable properties such as environmental friendliness, low cost, and low density. As a consequence, compared to nanoparticles and polymers, one-dimensional CNFs are more suitable for use as a barrier to prevent the irreversible self-accumulation of graphene nanosheet and agglomeration of the $\mathrm{MoS}_{2}$ nanosheet; moreover, they can be used as aqueous electrolyte nano-reservoirs, hierarchical nanostructure makers, and nano-spacers of RGO/ $\mathrm{MoS}_{2}$-based electrode materials for flexible supercapacitors.

In addition, CNFs prepared via 2, 2, 6, 6-tetramethylpiperidine-1-oxyl (TEMPO)-catalytic oxidation are a kind of nanometer fiber with high aspect ratio and a large number of COONa groups on their surface. This characteristic indicates that the CNFs hydrogel can be prepared by simply changing the concentration or the $\mathrm{pH}$ of the CNFs suspension and then drying it to form CNFs aerogels. Their aerogels are not only cheap, environmentally friendly, and biocompatible, but also have a porous tough skeleton structure, an extremely remarkable electrolyte-absorption property, ${ }^{33-36}$ an easy chemical-modified surface, and other advantages. Additionally, the hydrophilicity of CNFs in the aerogels can be conducive to the contact between the electrodes and electrolytes and also provide diffusion channels for the electrolyte ions; this consequently intensifies the property of the supercapacitors. ${ }^{7}$

In this study, we researched the free-standing, lightweight, and all-solid-state flexible supercapacitors with $\mathrm{CNFs} / \mathrm{MoS}_{2} / \mathrm{RGO}$ aerogel film electrodes prepared by a simple and environmentally friendly method (Fig. 1); these supercapacitors exhibit high specific capacitance and also display significantly high areal capacitance, areal power density, and energy density. These findings indicate that these all-solid-state flexible $\mathrm{CNFs} / \mathrm{MoS}_{2} /$ RGO nanohybrid aerogel film-based supercapacitors possess brilliant performance.

\section{Experimental}

\section{Materials}

Hardwood bleached kraft pulps in the never-dried wet state with $32 \%$ solid content was used. Tetramethylpiperidine-1-oxyl radical (TEMPO) and sodium ascorbate $\left(\mathrm{V}_{\mathrm{C}}-\mathrm{Na}\right)$ were provided by Aladdin Chemistry Co. Ltd. Sodium bromide ( $\mathrm{NaBr}$ ), sodium hypochlorite solution, sodium molybdate $\left(\mathrm{Na}_{2} \mathrm{MoO}_{4} \cdot 2 \mathrm{H}_{2} \mathrm{O}\right)$, L-cysteine, graphite powder, potassium peroxodisulfate $\left(\mathrm{K}_{2} \mathrm{~S}_{2} \mathrm{O}_{8}\right)$, phosphorus pentoxide $\left(\mathrm{P}_{2} \mathrm{O}_{5}\right)$, hydrogen peroxide $\left(\mathrm{H}_{2} \mathrm{O}_{2}\right)$, and polyvinyl alcohol (PVA) were bought from Sinopharm Chemical Reagent Beijing Co. Ltd. Potassium permanganate $\left(\mathrm{KMnO}_{4}\right)$ was purchased from Beijing Beihua Fine Chemical Products Co. Ltd. Concentrated sulfuric acid was obtained from Beijing Chemical Works. All the chemicals were used without further purification.

\section{Preparation of $\mathrm{CNFs} / \mathrm{MoS}_{2} / \mathrm{RGO}$ aerogels}

CNFs, $\mathrm{MoS}_{2}$, and GO nanosheets were synthesized according to the literature methodology reported by Isogai, Meng, and Tung, 
respectively. ${ }^{37-39}$ The detailed preparation process is provided in the ESI. $\dagger$ Fig. 1 shows the preparation process of the CNFs/ $\mathrm{MoS}_{2} / \mathrm{RGO}$ aerogels that has been described in brief as follows: (i) the prepared $\mathrm{MoS}_{2}(40 \mathrm{mg})$ nanosheets were dispersed in CNFs suspension (50 g, 0.2 wt\%) via sonication. The GO suspension (12 g, $0.5 \mathrm{wt} \%$ ) was added to the $\mathrm{CNFs} / \mathrm{MoS}_{2}$ suspension and then sonicated until a uniform $\mathrm{CNFs} / \mathrm{MoS}_{2} / \mathrm{GO}$ co-suspension was formed. The weight percentages of CNFs, $\mathrm{MoS}_{2}$, and GO were 50\%, 20\%, and 30\%, respectively. (ii) Excess $\mathrm{V}_{\mathrm{C}}-\mathrm{Na}$ was added to the abovementioned co-suspension and stirred for 3 min until $\mathrm{V}_{\mathrm{C}}-\mathrm{Na}$ was completely dissolved in the cosuspension. The resulting $\mathrm{CNFs} / \mathrm{MoS}_{2} / \mathrm{GO}-\left(\mathrm{V}_{\mathrm{C}}-\mathrm{Na}\right)(10 \mathrm{~g})$ hybrid suspension was transferred into a $20 \mathrm{ml}$ glass mold that was then placed under a hydrochloric acid atmosphere for 4 hours. After this, the $\mathrm{CNFs} / \mathrm{MoS}_{2} / \mathrm{GO}-\left(\mathrm{V}_{\mathrm{C}}-\mathrm{Na}\right)$ suspension was gradually transformed into a $\mathrm{CNFs} / \mathrm{MoS}_{2} / \mathrm{GO}-\left(\mathrm{V}_{\mathrm{C}}-\mathrm{Na}\right)$ nanohybrid hydrogel under the action of hydrogen bonding. (iii) The CNFs/ $\mathrm{MoS}_{2} /$ RGO nanohybrid hydrogels formed after the CNFs/MoS $/$ $\mathrm{GO}-\left(\mathrm{V}_{\mathrm{C}}-\mathrm{Na}\right)$ nanohybrid hydrogels were stored at $80{ }^{\circ} \mathrm{C}$ for $24 \mathrm{~h}$. Next, the CNFs/MoS $/$ RGO nanohybrid hydrogels were replaced with $\mathrm{CNFs} / \mathrm{MoS}_{2} / \mathrm{RGO}$ nanohybrid alcogels following which the nanohybrid hydrogels were washed with a large amount of deionized water. (iv) The CNFs/MoS $2 /$ RGO nanohybrid aerogels were prepared from nanohybrid alcogels by supercritical $\mathrm{CO}_{2}$ drying. Fig. S1† displays the image of the $\mathrm{CNFs} / \mathrm{MoS}_{2} / \mathrm{RGO}$ nanohybrid aerogel.

\section{Fabrication of the flexible all-solid-state supercapacitors}

The $\mathrm{H}_{2} \mathrm{SO}_{4}$ /polyvinyl alcohol (PVA) gel is chosen as an electrolyte. ${ }^{39}$ The process of preparation of $\mathrm{CNFs} / \mathrm{MoS}_{2} / \mathrm{RGO}$ nanohybrid aerogel electrodes and fabrication of the all-solid-state flexible supercapacitors are also shown in Fig. 1. The CNFs/ $\mathrm{MoS}_{2} /$ RGO nanohybrid aerogels were compressed into aerogel films under a pressure of 0.5 MPa (Fig. S2 $\dagger$ ), and then, the edge of one side of the compressed film was pasted with aluminum foil using a silver paste to promote the electrical contact of the electrode. After this, two pieces of the aerogel films $(20 \mathrm{~mm} \times$ $10 \mathrm{~mm}$ ) were dipped in the hot $\mathrm{H}_{2} \mathrm{SO}_{4} / \mathrm{PVA}$ gel electrolyte for 15 min, which was also simultaneously used as a separator ${ }^{39}$ and then taken out to be placed in the fume hood for about $6 \mathrm{~h}$ to vaporize the excessive water at room temperature. Finally, the two pieces of the resulting nanohybrid aerogel films were assembled into a flexible all-solid-state supercapacitor under a pressure of $0.5 \mathrm{MPa}$ for $15 \mathrm{~min}$, as shown in the Fig. S3. $\dagger$

\section{Nanohybrid characterization}

The microstructure and morphology of the samples were characterized using a Hitachi S-4800 field-emission-gun scanning electron microscope (SEM) at $5 \mathrm{kV}$ and a JEM-200CX transmission electron microscope (TEM). The high resolution transmission electron microscopy (HRTEM) observations were obtained using a Tecnai F20 TEM at $200 \mathrm{kV}$ acceleration voltage, and the chemical composition was investigated by energy dispersive X-ray spectroscopy (EDS). X-ray diffraction (XRD) analysis was conducted via a Bruker AXS D8 Discover diffractometer (Germany) using $\mathrm{Cu}-\mathrm{K} \alpha$ radiation $(\lambda=1.5406 \AA)$. The $2 \theta$ used in the measurements ranged from 10 to $30^{\circ}$. Nitrogen sorption measurements were conducted using an ASAP 2010 (Micromeritics, USA) to obtain pore properties. X-ray photoelectron spectroscopy (XPS) of the samples was carried out using a PHI 5300 Photoelectron Spectrometer (Perkin Elmer Instruments Co. Ltd., USA). Fourier transform infrared spectroscopy (FT-IR) was conducted using a Magna-IR 750 spectrophotometer.

\section{Electrochemical characterization}

The electrochemical performances of the all-solid-state flexible supercapacitors were assessed using a CHI 660D electrochemical workstation, and all the measurements were executed in air at room temperature. Cyclic voltammograms (CV) were measured from 0 to $1 \mathrm{~V}$ at the scan rates of 2, 5, 10, 50, 100, 200, and $400 \mathrm{mV} \mathrm{s}^{-1}$. The galvanostatic charge-discharge (GCD) property was determined at the current densities of $0.5,1,2.5,5$, 7.5 , and $10 \mathrm{~mA} \mathrm{~cm}^{-2}$ with the cut-off voltages from 0 to $1 \mathrm{~V}$. The electrochemical impedance spectroscopy (EIS) was performed using a sinusoidal signal of $5 \mathrm{mV}$ in the frequency range from $0.01 \mathrm{~Hz}$ to $100 \mathrm{kHz}$ under an open circuit potential. Cyclic stabilities were characterized using GCD measurements for over 5000 cycles at the current density of $5 \mathrm{~mA} \mathrm{~cm}^{-2}$.

\section{Results and discussion}

\section{Characterization of the $\mathrm{CNFs} / \mathrm{MoS}_{2} / \mathrm{RGO}$ nanohybrid aerogel electrodes}

Fig. 2 illustrates the SEM images of the CNFs aerogel, pristine $\mathrm{MoS}_{2}$ powder, CNFs/MoS ${ }_{2}$, and $\mathrm{CNFs} / \mathrm{MoS}_{2} / \mathrm{RGO}$ nanohybrid aerogel and the TEM images of CNFs, $\mathrm{MoS}_{2}, \mathrm{CNFs} / \mathrm{MoS}_{2}$, and CNFs/ $\mathrm{MoS}_{2} / \mathrm{GO}$ suspension. As shown in Fig. 2a, c, and d, all the aerogels possess a highly interconnected $3 \mathrm{D}$ porous structure. Fig. $2 \mathrm{~b}$ and f clearly show that the pristine $\mathrm{MoS}_{2}$ has a relatively uniform spherical morphology with a mean diameter of about $300 \mathrm{~nm}$, and these spheres are built up with many $\mathrm{MoS}_{2}$ nanosheets; this indicates that the as-prepared $\mathrm{MoS}_{2}$ nanosheets are also easy to reunite and form a flower-like structure. As shown in Fig. 2c and g, the $\mathrm{MoS}_{2}$ nanosheets are attached along the CNFs fibrils and uniformly dispersed. Fig. $\mathrm{S} 4 \uparrow$ shows $\mathrm{MoS}_{2}$ with different weight percentages in the CNFs/MoS 2 aerogels. When the weight percentages of $\mathrm{MoS}_{2}$ in $\mathrm{CNFs} / \mathrm{MoS}_{2}$ are more than $30 \%, \mathrm{MoS}_{2}$ nanosheets begin to accumulate and stack together on the CNFs network structure. Therefore, a reasonable amount of CNFs can effectively prevent the aggregation of the $\mathrm{MoS}_{2}$ nanosheets. In Fig. 2d, a number of irregular pores and apertures can be observed in the CNFs/ $\mathrm{MoS}_{2}$ /RGO nanohybrid aerogel, and the CNFs and RGO nanosheets form a stable 3D porous network structure; moreover, the $\mathrm{MoS}_{2}$ flake is evenly embedded in these aerogel sheets and cell structures such that more edge sites are exposed. The SEM image of the $\mathrm{MoS}_{2} / \mathrm{RGO}$ hybrid aerogel without CNFs is shown in Fig. S5. $\dagger$ As can be seen from the figure, RGO shows a packed structure with little apertures on the nanosheets, and $\mathrm{MoS}_{2}$ seriously stack together on the RGO nanosheets. Thus, the presence of CNFs prevent the aggregation of $\mathrm{MoS}_{2}$ and RGO, and these three form the unique architecture that not only 

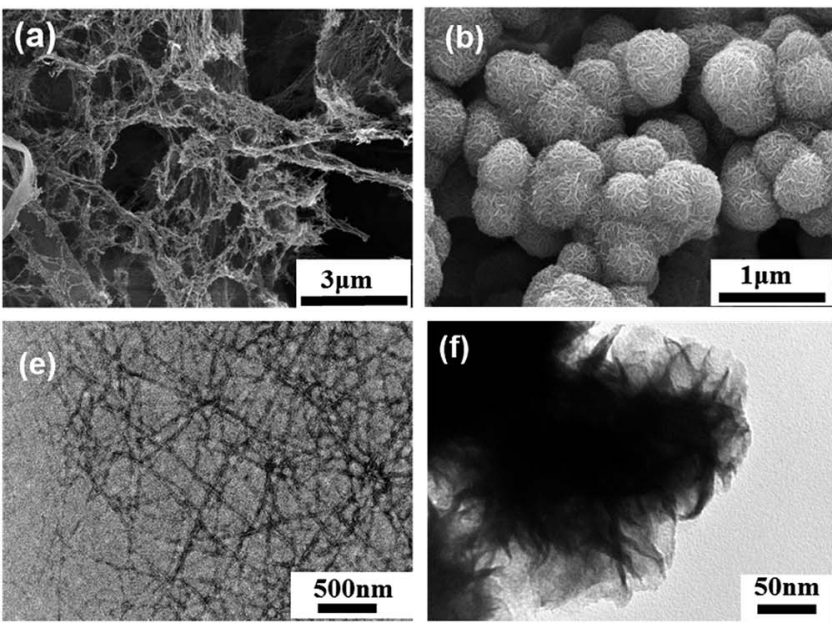
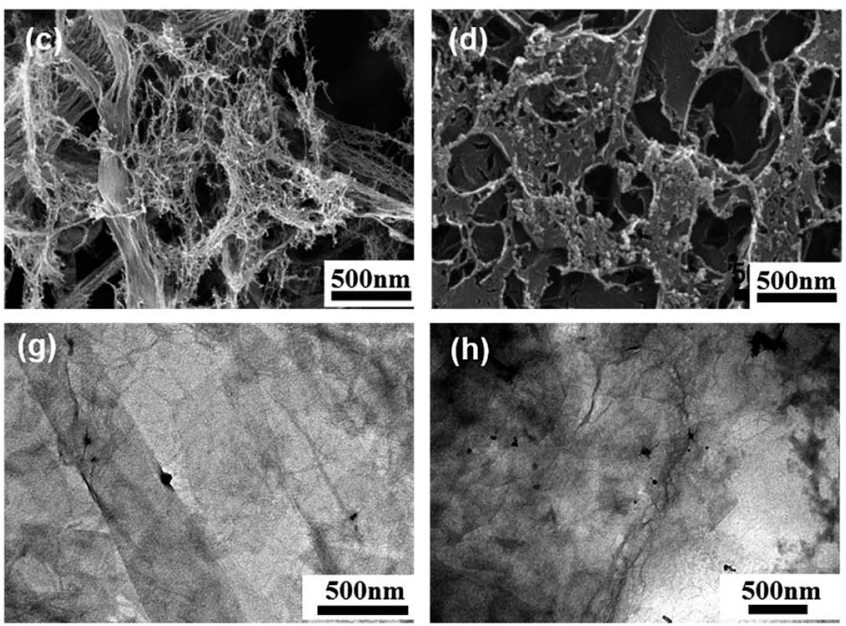

Fig. 2 SEM images of the CNFs aerogel (a), $\mathrm{MoS}_{2}$ powder (b), CNFs/MoS 2 nanohybrid aerogel (c), and CNFs/MoS $2 / \mathrm{RGO}$ nanohybrid aerogel (d); TEM images of the CNFs suspension (e), $\mathrm{MoS}_{2}$ suspension (f), CNFs/MoS 2 hybrid suspension (g), and CNFs/MoS $2 / \mathrm{GO}$ hybrid suspension (h).

greatly reduces the diffusion length and provides extra continuous conductive pathways between different layers to improve the specific surface area, but also ensure that the CNFs is a steady dispersant of $\mathrm{MoS}_{2}$ and RGO. Fig. S6† shows the optical images of the aqueous dispersion of $\mathrm{CNFs} / \mathrm{MoS}_{2} / \mathrm{GO}, \mathrm{CNFs} /$ $\mathrm{MoS}_{2}$, and CNFs suspension. From the image, it can be observed that GO and $\mathrm{MoS}_{2}$ can be well dispersed in the CNFs suspension after sonication and remain stable at room temperature for several months. This phenomenon can also be confirmed by the TEM images (Fig. 2e, g and h).
To more clearly confirm the dispersion of $\mathrm{MoS}_{2}$ in the CNFs/ $\mathrm{MOS}_{2} /$ RGO hybrid, TEM EDS analysis was carried out. The EDS spectrum of the CNFs/MoS $/$ /RGO hybrid (Fig. 3b) shows characteristic peaks for $\mathrm{C}, \mathrm{O}, \mathrm{Mo}$, and $\mathrm{S}$, which prove the coexistence of these elements. Moreover, the atomic percentage of the Mo element and S element in the $\mathrm{CNFs} / \mathrm{MoS}_{2} / \mathrm{RGO}$ hybrid is about $2.62 \%$ and $5.65 \%$, respectively, corresponding to the Mo to $\mathrm{S}$ molar ratio of about 1-2. From the HRTEM image shown in the inset of Fig. 3b, 4-7 layers of $\mathrm{MoS}_{2}$ nanosheets can be clearly observed. The interlayer spacing of $\mathrm{MoS}_{2}$ nanosheets is about
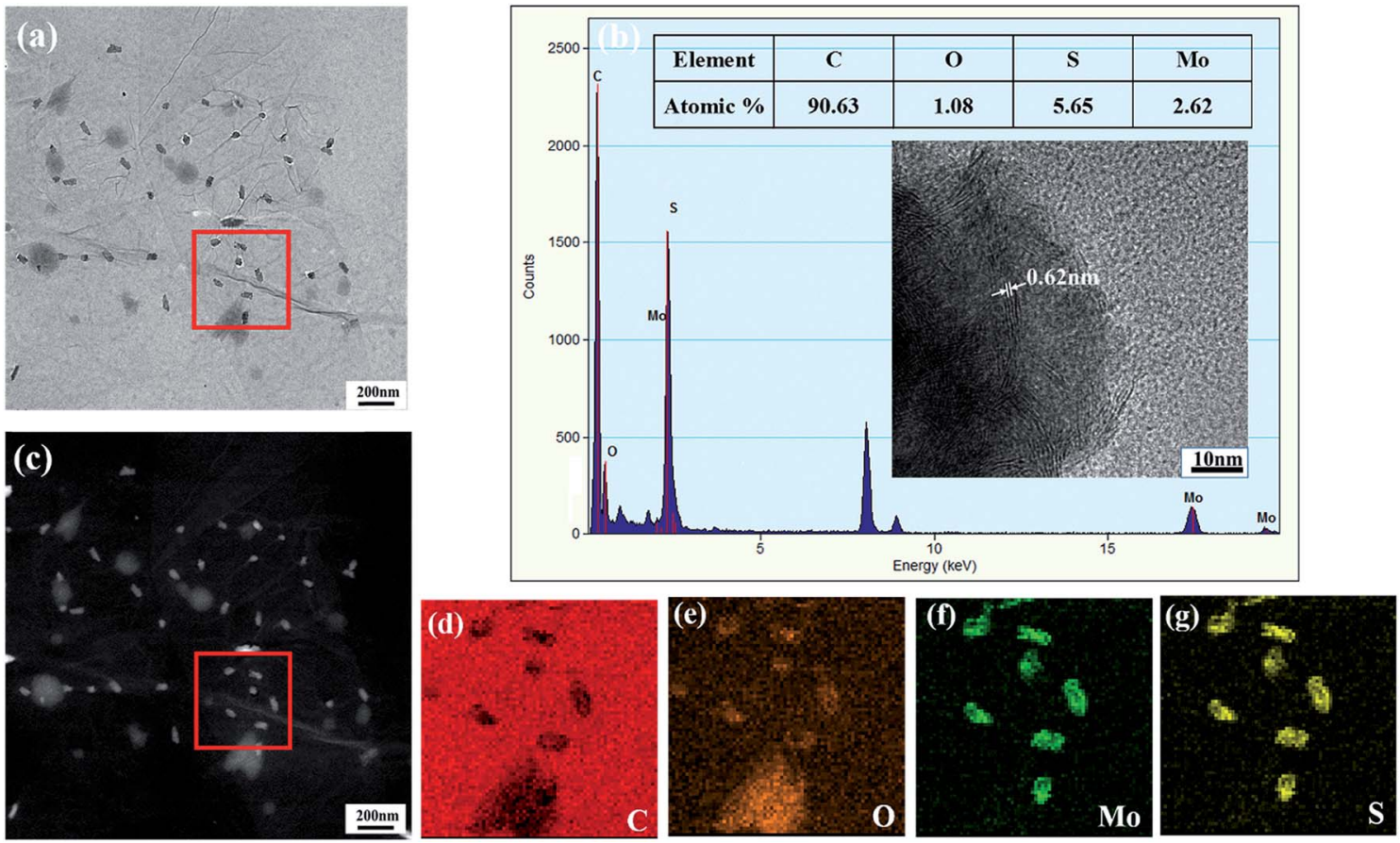

Fig. 3 TEM EDS analysis of the CNFs/MoS $/$ RGO hybrid. (a) The magnified TEM image of the CNFs/MoS $/$ /RGO hybrid suspension. (b) EDS spectra and the atomic percentage of the element. The inset shows the HRTEM image of the CNFs/MoS $2 / R G O$ hybrid. (c) Dark field of TEM image. (d-g) The corresponding EDS elemental mappings of the CNFs/MoS $2 / R G O$ hybrid. 

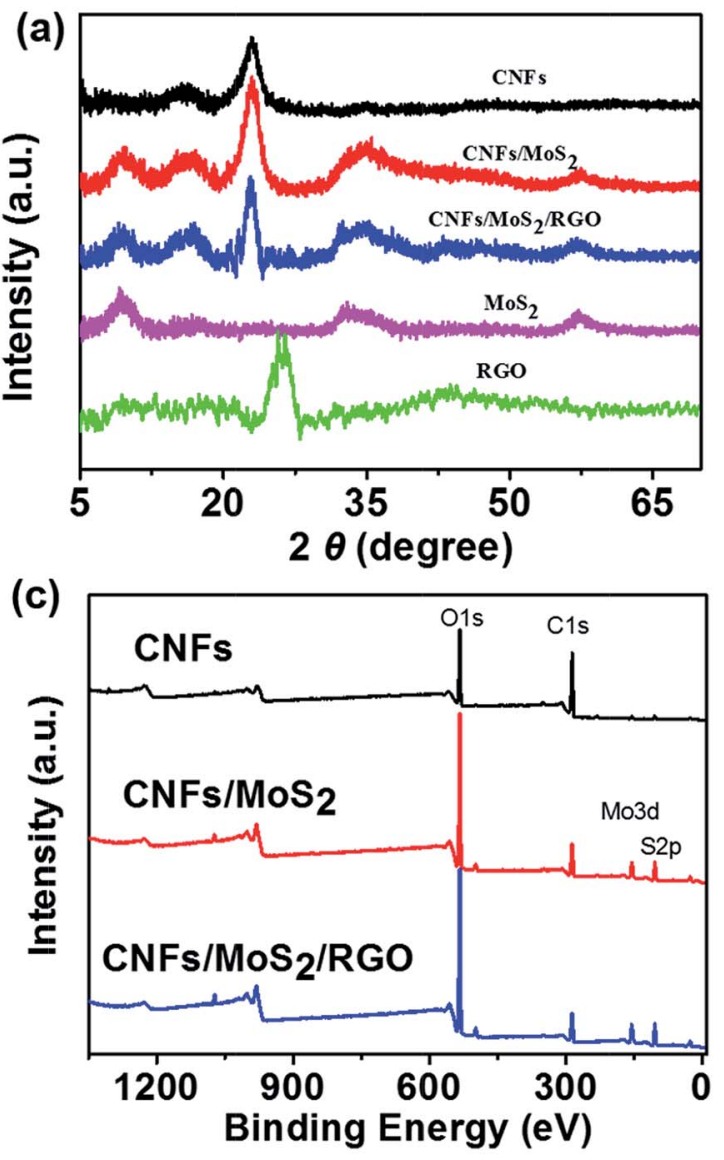

(b)

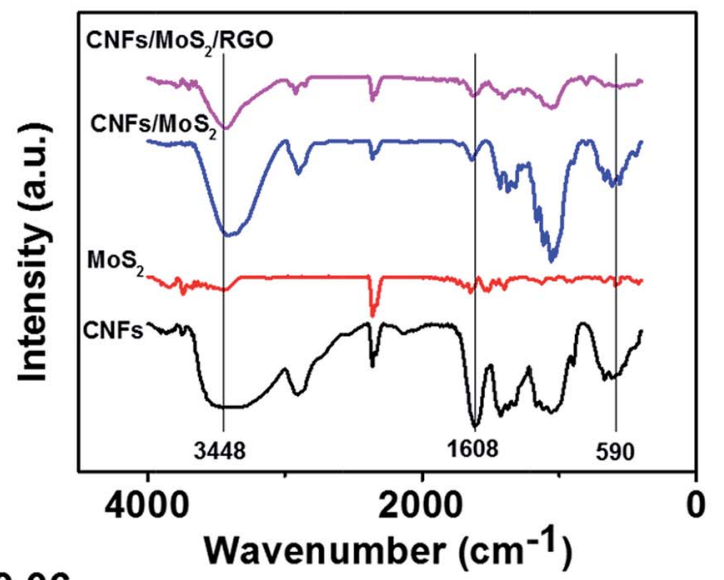

(d)

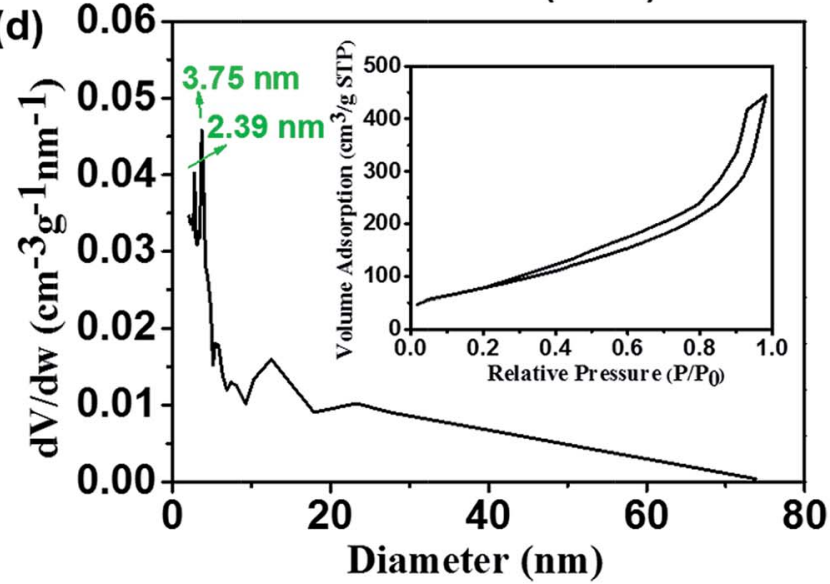

Fig. 4 XRD patterns (a), FT-IR (b), XPS (c), and BJH pore size distribution curves (d) of samples. The inset of (d) shows the $\mathrm{N}_{2}$ adsorption/ desorption isotherm.

$0.62 \mathrm{~nm}$ that corresponds to the (002) lattice of hexagonal $\mathrm{MoS}_{2}$, which also agrees with the next XRD analysis. The EDS elemental mappings of the CNFs/ $\mathrm{MoS}_{2} /$ RGO hybrid (Fig. 3d-g) display the elemental distribution of $\mathrm{C}, \mathrm{O}, \mathrm{Mo}$, and $\mathrm{S}$, which also prove the coexistence of these elements and further confirm that the $\mathrm{MoS}_{2}$ nanosheets are evenly embedded in the CNFs/ $\mathrm{MoS}_{2} / \mathrm{RGO}$ hybrid aerogel sheets and cell structures.

Fig. 4a shows the X-ray diffraction (XRD) patterns of RGO and $\mathrm{MoS}_{2}$ nanosheets, CNFs, CNFs/MoS 2 , and CNFs/MoS 2 /RGO aerogels. The XRD pattern of the CNFs aerogel shows two overlapped peaks at $2 \theta=14-18^{\circ}$, in accordance with the (101) and (101) lattice planes of the cellulose I, and an obvious peak at $2 \theta=22.5^{\circ}$, attributed to the (002) lattice planes of the cellulose I crystalline structure..$^{35,40,41}$ The pattern of the pure $\mathrm{MoS}_{2}$ shows diffraction peaks at $2 \theta=14^{\circ}$ and $59^{\circ}$, which can be attributed to the (002) and (110) planes, respectively, and also shows three overlapping peaks at $2 \theta=33-40^{\circ}$, which can be readily indexed to the (101), (102), and (103) planes. ${ }^{42}$ The XRD pattern of the $\mathrm{CNFs} / \mathrm{MoS}_{2}$ nanohybrid aerogel presents crystalline peaks similar to the combination of the peaks obtained from CNFs and $\mathrm{MoS}_{2}$; this reveals that no additional crystalline order has been introduced into the hybrid. The XRD pattern of the CNFs/ $\mathrm{MoS}_{2} /$ RGO nanohybrid aerogel displays the peaks of CNFs and $\mathrm{MoS}_{2}$, whereas no peak at $25.5^{\circ}$ corresponding to the (002) lattice planes of RGO is observed. This result demonstrates that the CNFs can effectively prevent the $\pi-\pi$ stacking of graphene nanosheets. ${ }^{43}$

The FT-IR measurement was implemented to obtain the bending and stretching vibrations of the functional groups present in the samples. As shown in Fig. 4 b, the weak peaks at about $590 \mathrm{~cm}^{-1}$ in the FT-IR spectra of $\mathrm{MoS}_{2}, \mathrm{CNFs} / \mathrm{MoS}_{2}$, and $\mathrm{CNFs} / \mathrm{MoS}_{2} / \mathrm{RGO}$ are assigned to the Mo-S vibration. ${ }^{44}$ The peaks at $1608 \mathrm{~cm}^{-1}$, consistent with the -COO- stretching vibration, can confirm the electronegativity of the CNFs, resulting in the uniform dispersion of CNFs in water. The bond at $3448 \mathrm{~cm}^{-1}$ appears in all the CNFs, $\mathrm{MoS}_{2}, \mathrm{CNFs} / \mathrm{MoS}_{2}$, and $\mathrm{CNFs} / \mathrm{MoS}_{2} / \mathrm{RGO}$ spectra, which is mainly attributed to the stretching vibrations of the $\mathrm{O}-\mathrm{H}$ bonds. The differentiation of the intensity of the $\mathrm{OH}$ vibration showed that the free hydroxy groups decrease after the RGO is added. Therefore, the results from the FTIR spectra demonstrate that $\mathrm{MoS}_{2}$ and RGO have been tightly incorporated into the CNFs backbone.

The surface chemistry of the CNFs, CNFs/MoS 2 , and CNFs/ $\mathrm{MoS}_{2} / \mathrm{RGO}$ aerogel electrodes was further investigated using XPS spectra. As can be seen from Fig. 4c, the survey scan spectra of the CNFs $/ \mathrm{MoS}_{2} / \mathrm{RGO}$ nanohybrid aerogel shows the characteristic absorption peaks of O 1s, C 1s, S 2p, Mo 3d, which further confirms the stable blend of CNFs, $\mathrm{MoS}_{2}$, and RGO. 

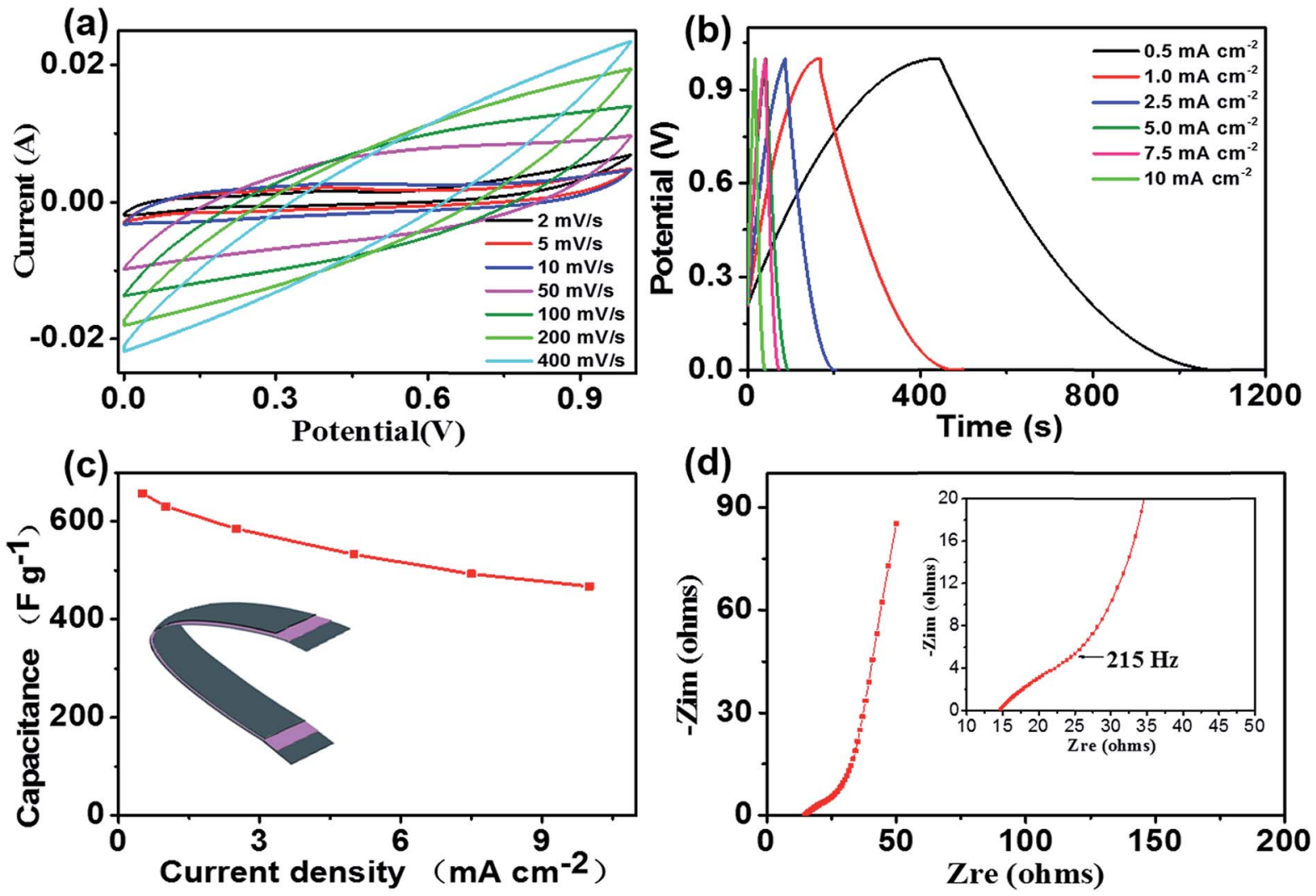

Fig. 5 (a) Typical CV curves of the CNFs/MoS $/$ RGO nanohybrid aerogel supercapacitor at different scan rates. (b) Typical galvanostatic chargedischarge curves of the $\mathrm{CNFs} / \mathrm{MoS}_{2} / \mathrm{RGO}$ nanohybrid aerogels supercapacitor at different current densities. (c) The specific electrode capacitance of the CNFs/MoS $/$ RGO nanohybrid aerogels supercapacitor as a function of the current density. Inset shows the image of the all-solidstate flexible supercapacitor. (d) Nyquist impedance plots of the supercapacitor. Inset shows a magnified view of the high frequency region.

The specific surface area and porous structure of the samples were characterized by measuring the $\mathrm{N}_{2}$ adsorption-desorption isotherms. From Fig. S7a $\uparrow$ and the inset of Fig. 4 d, it can be observed that the isotherm curves have the characteristic of a type IV isothermal curve; this indicates that the CNFs aerogel, pure $\mathrm{MoS}_{2}$, RGO aerogel, and CNFs/MoS $/$ RGO hybrid aerogel possess a large quantity of mesopores. The pore size distribution (in the range of $0-74 \mathrm{~nm}$ ) of the $\mathrm{CNFs} / \mathrm{MoS}_{2} / \mathrm{RGO}$ hybrid aerogel (Fig. 4d) measured by the Barrett-Joyner-Halenda (BJH) method shows that the distribution is centered at $2.39 \mathrm{~nm}$ and $3.75 \mathrm{~nm}$, which confirm the mesoporous characteristics of $\mathrm{CNFs} / \mathrm{MoS}_{2} / \mathrm{RGO}$ hybrid aerogel. The Brunauer-Emmett-Teller (BET) specific surface area of the $\mathrm{CNFs} / \mathrm{MoS}_{2} / \mathrm{RGO}$ hybrid aerogel is about $287.2 \mathrm{~m}^{2} \mathrm{~g}^{-1}$. Fig. S7c and $\mathrm{d} \dagger$ show the pore size distribution of the CNFs aerogel, pure $\mathrm{MoS}_{2}$, and RGO aerogel. As can be seen from the figure, these three are mainly characteristic of mesopores and the distribution is as follows: at CNFs aerogel: $2.30 \mathrm{~nm}-2.81 \mathrm{~nm}$ and $3.80 \mathrm{~nm}$, at RGO aerogel: $2.1 \mathrm{~nm}$, and at pure $\mathrm{MoS}_{2}: 2.06 \mathrm{~nm}$. It is worth noting that $\mathrm{MoS}_{2}$ shows another distribution that is centered at $1.71 \mathrm{~nm}$. This illustrates that $\mathrm{MoS}_{2}$ simultaneously possesses micropores and mesopores, which may originate from the void spaces among the flakes. Their specific surface areas are as follows: CNFs aerogel: $264 \mathrm{~m}^{2} \mathrm{~g}^{-1}$, RGO aerogel: $368.6 \mathrm{~m}^{2} \mathrm{~g}^{-1}$, and pure $\mathrm{MoS}_{2}: 13.3 \mathrm{~m}^{2} \mathrm{~g}^{-1}$. Therefore, by comparing the pore size distribution of all the samples and according to the SEM images, the mesopores can be attributed to the 3D porous net-like framework provided by the CNFs and we can make sure that the mesopores are mainly produced by the hierarchical nanostructure of the solid walls.

\section{Electrochemical characteristics of all-solid-state flexible supercapacitors}

For showing the electrochemical performance of the CNFs/ $\mathrm{MoS}_{2} / \mathrm{RGO}$ nanohybrid aerogels, the compressed CNFs/MoS $/$ RGO nanohybrid aerogels films are used to manufacture allsolid-state flexible supercapacitors (Fig. S3†). The electrolyte of the supercapacitors is $\mathrm{H}_{2} \mathrm{SO}_{4}$ /polyvinyl alcohol (PVA) gel, ${ }^{39}$ which plays the function of a separator as well.

Fig. 5a displays the cyclic voltammetry (CV) curves of the supercapacitor device at the scan rates of $2-400 \mathrm{mV} \mathrm{s}^{-1}$ in the potential window ranging from 0 to $1 \mathrm{~V}$. The meristic and quasirectangular CV curves suggest the typical electrical double layer capacitive behaviour with quite fast current response upon voltage reversal. Moreover, the $\mathrm{CV}$ curve still maintains its shape even at a high scan rate of $400 \mathrm{mV} \mathrm{s}^{-1}$; this demonstrates the good high-rate performance of the supercapacitor. The specific electrode capacitance $\left(C_{g}\right)$ of the supercapacitor, which is calculated from the cyclic voltammogram curves, is about $916.43 \mathrm{~F} \mathrm{~g}^{-1}$ (based on the total mass of $\mathrm{RGO} / \mathrm{MoS}_{2}$ ) at a scan 

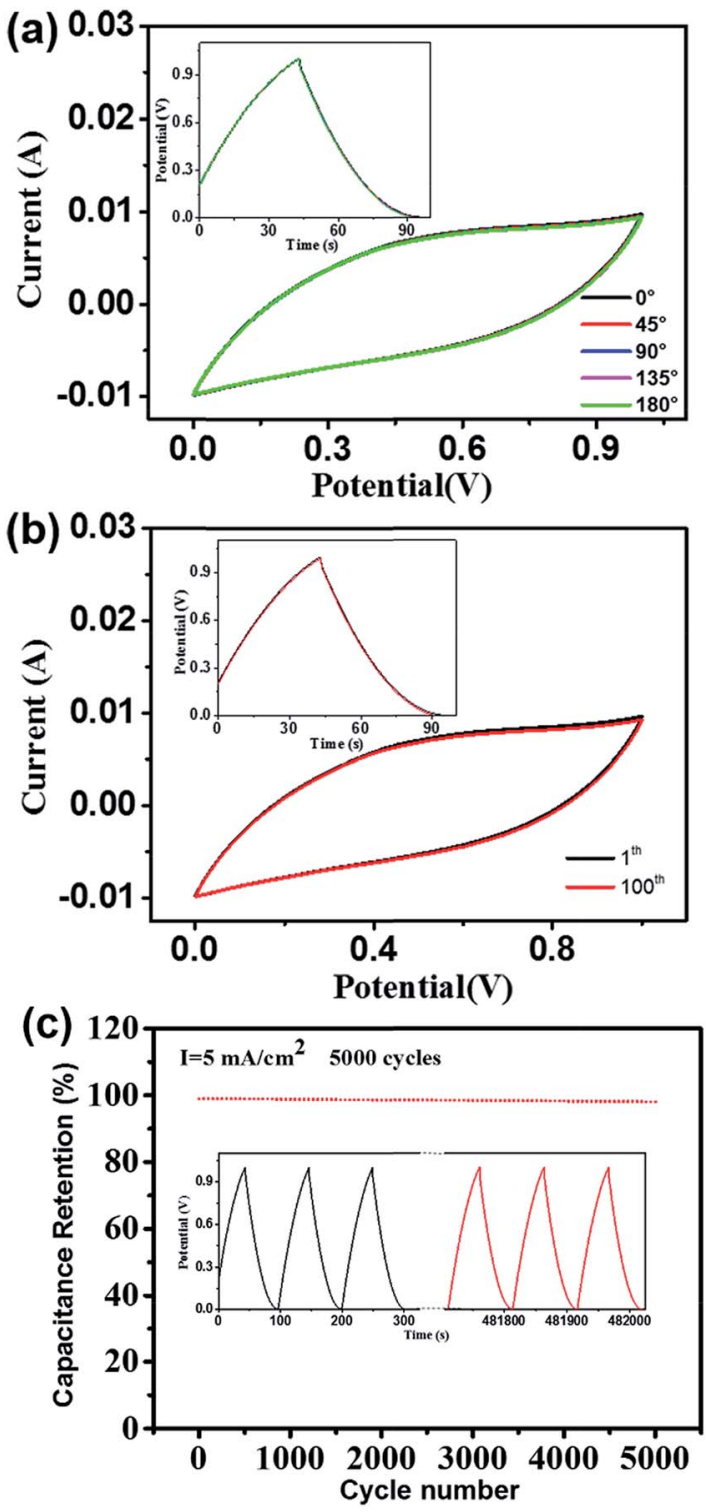

Fig. 6 (a) CV curves of the supercapacitor at different bending angles with a scan rate of $50 \mathrm{mV} \mathrm{s}^{-1}$. Inset shows the GCD curves at different bending angles with a current density of $5 \mathrm{~mA} \mathrm{~cm}^{-2}$. (b) $C V$ curves before and after 100 bending cycles for supercapacitor at $50 \mathrm{mV} \mathrm{s}^{-1}$. Inset shows the GCD curves before and after 100 bending cycles at $5 \mathrm{~mA} \mathrm{~cm}^{-2}$. (c) Cycling stability of the supercapacitor over 5000 cycles at the current density of $5 \mathrm{~mA} \mathrm{~cm}^{-2}$; the inset shows the galvanostatic charge-discharge curve.

rate of $2 \mathrm{mV} \mathrm{s}^{-1}$, which is much higher than the $C_{\mathrm{g}}$ of many other graphene and $\mathrm{MoS}_{2}$-based electrode materials measured in liquid electrolytes (Table $\mathrm{S} 1 \dagger$ ). In addition, the areal capacitance (CV) of the supercapacitor is $458.21 \mathrm{mF} \mathrm{cm}^{-2}$, which is much higher than that of other types of recently reported flexible supercapacitor devices (Table S2 $\dagger$ ).

Galvanostatic charge-discharge (GCD) curves of the CNFs/ $\mathrm{MoS}_{2} / \mathrm{RGO}$ nanohybrid aerogels supercapacitor at different current densities with the voltage between 0 and $1 \mathrm{~V}$ are shown in Fig. 5b. The curves display a perfect linear profile, and all the charge curves are ideal symmetrical with their corresponding discharge curves within the potential window. This demonstrates that the supercapacitors have remarkable capacitive performance and rapid current-voltage response. The $C_{\mathrm{g}}$, which is calculated from the discharge curves at different current densities, is displayed in Fig. 5c. As can be seen from the figure, the supercapacitor has a specific capacitance of $657.7 \mathrm{~F} \mathrm{~g}^{-1}$ at a current density of $0.5 \mathrm{~mA} \mathrm{~cm} \mathrm{~cm}^{-2}$. Moreover, when the current density is increased from $0.5 \mathrm{~mA} \mathrm{~cm}^{-2}$ to $10 \mathrm{~mA} \mathrm{~cm}^{-2}$, the $C_{\mathrm{g}}$ $\left(468 \mathrm{~F} \mathrm{~g}^{-1}\right)$ is still maintained at $71.2 \%$ of the initial capacitance; this indicates that the CNFs/ $\mathrm{MoS}_{2} / \mathrm{RGO}$ nanohybrid aerogel supercapacitors have a favourable capacitance retention capability. The excellent electrochemical performances of the supercapacitors can be ascribed to several factors: first, the onedimensional CNFs available prevents the aggregation of graphene nanosheets and agglomeration of $\mathrm{MoS}_{2}$ nanosheets, and the CNFs with superior hydrophilic characteristics form a highly open, continuous pore structure that displays a high specific surface energy, allows full contact between the electrodes and the electrolyte, thus providing diffusion channels for the electrolyte ions; second, $\mathrm{MoS}_{2}$, which is evenly embedded in the $\mathrm{CNF} / \mathrm{MoS}_{2} / \mathrm{RGO}$ nanohybrid aerogel sheets and 3D porous network structures, not only greatly reduces the length of the diffusion path that both electrons and ions have to transfer over during the charge/discharge process, but also provides more extra continuous conductive pathways between different layers of hybrid nanosheets; third, the excellent interfacial contact between $\mathrm{MoS}_{2}$ and RGO obviously facilitates the transport of electrons throughout the whole electrode matrix. This unique architecture of the CNFs/ $\mathrm{MoS}_{2} / \mathrm{RGO}$ nanohybrid aerogel, which can be seen from the SEM images (Fig. 2d), enables the nanohybrid electrode to simultaneously have a large specific surface and rapid electron and ion transport; thus, the supercapacitor shows excellent electrochemical performance.

A superior electrochemical capacitor electrode material should possess high capacitance and low electronic resistance. The electrochemical impedance spectroscopy (EIS) analysis has been approbated as one of the primary methods of examining the basic behaviour of electrode materials for supercapacitors. ${ }^{45}$ Fig. $5 \mathrm{~d}$ shows the Nyquist plots of the fabricated supercapacitor using a sinusoidal signal of $5 \mathrm{mV}$ in the frequency range from $0.01 \mathrm{~Hz}$ to $100 \mathrm{kHz}$ under an open circuit potential. At high frequency, the equivalent series resistance $\left(R_{\mathrm{ESR}}\right)$ value acquired from the intercept of the Nyquist diagram with the real axis is about $14.6 \Omega$. Additionally, the absence of a semicircle is probably attributed to the charge stored primarily via a nonfaradaic process. In the medium frequency region, the projected length obtained from the Warburg-type line with a slope of approximately $45^{\circ}$ on the real axis is used to assess the diffusion process of electrolyte ions in porous electrodes. ${ }^{46-48}$ As seen from the figure, the Warburg-type line is relatively short, revealing the fast electrolyte ion diffusion in the CNFs/ $\mathrm{MoS}_{2} /$ RGO nanohybrid aerogel electrode materials, which corresponds with the results acquired from the galvanostatic chargedischarge curves. The $\mathrm{CNFs} / \mathrm{MoS}_{2} / \mathrm{RGO}$ nanohybrid aerogel electrode materials are easy to re-expand in an aqueous electrolyte because of even dispersion throughout the whole electrode and the remarkable hydrophilicity of the CNFs. These 
CNFs can serve as nano-reservoirs of the aqueous electrolyte, and a producer of highly continuous open pores, which can obviously reduce the diffusion distance of electrolyte ions. At low frequency, the supercapacitor device displays a straight and nearly vertical line, demonstrating ideal capacitance characteristics. The knee frequency of the supercapacitor is $215 \mathrm{~Hz}$, as illustrated in the inset in Fig. 5d of the enlarged plot of the high-frequency area, which indicates that the supercapacitor displays a pure capacitive behaviour, and most of the stored energy is available. Moreover, these supercapacitors display a much high areal energy density of $45.7 \mu \mathrm{W} \mathrm{h} \mathrm{cm} \mathrm{cm}^{-2}\left(22.8 \mathrm{~W} \mathrm{~h} \mathrm{~kg}^{-1}\right)$ at an areal power density of $8.56 \mathrm{~mW} \mathrm{~cm}^{-2}\left(4.3 \mathrm{~kW} \mathrm{~kg}^{-1}\right)$. This demonstrates that the CNFs/ $\mathrm{MoS}_{2} /$ RGO nanohybrid aerogel is a promising electrode material for all-solid-state flexible supercapacitors.

The electrochemical stability in the bent state is also an important performance criterion for all-solid-state flexible supercapacitors. Fig. 6a shows the CV behaviour of the supercapacitor tested at a scan rate of $50 \mathrm{mV} \mathrm{s}^{-1}$ and the GCD at a current density of $5 \mathrm{~mA} \mathrm{~cm} \mathrm{~cm}^{-2}$ (the inset) under different bending angles. As seen from the figure, the bending has almost no negative effect on the electrochemical performance. Furthermore, the shape of the CV curve and specific capacitance of a supercapacitor (obtained from the GCD of inset in Fig. 6b) do not change obviously after withstanding 100 bending cycles (Fig. 6b); this indicates that the supercapacitors have good flexibility. ${ }^{9}$ Additionally, Fig. 6c shows that the flexible supercapacitor exhibits excellent cyclic stability, with more than $98 \%$ specific capacitance retention after 5000 charge-discharge cycles at the current density of $5 \mathrm{~mA} \mathrm{~cm}^{-2}$, and the SEM images show that the $\mathrm{CNFs} / \mathrm{MoS}_{2} / \mathrm{RGO}$ aerogel electrode infiltrated with the $\mathrm{H}_{2} \mathrm{SO}_{4}$ /PVA gel electrolyte has no apparent microstructure change after 5000 charge-discharge cycles (Fig. S8†).

\section{Conclusions}

In summary, the flexible all-solid-state supercapacitors employing compressed $\mathrm{CNFs} / \mathrm{MoS}_{2} / \mathrm{RGO}$ nanohybrid aerogels as electrode material exhibit excellent performance: the specific capacitance, areal power density, and energy density are $916.42 \mathrm{~F} \mathrm{~g}^{-1}$ (458.2 $\left.\mathrm{mF} \quad \mathrm{cm}^{-2}\right), 8.56 \mathrm{~mW} \quad \mathrm{~cm}^{-2}\left(4.3 \mathrm{~kW} \mathrm{~kg}^{-1}\right)$, and $45.7 \mu \mathrm{W} \mathrm{h} \mathrm{cm}{ }^{-2}\left(22.8 \mathrm{~W} \mathrm{~h} \mathrm{~kg}^{-1}\right)$, respectively. Additionally, the flexible supercapacitor exhibits remarkable cyclic stability, with more than $98 \%$ specific capacitance retention after 5000 chargedischarge cycles. The resulting $\mathrm{CNFs} / \mathrm{MoS}_{2} / \mathrm{RGO}$ nanohybrid aerogels as electrode materials in our study display a superior electrochemical performance, excellent bending stability, low cost, and environmental friendliness, which may be a potential electrode material for flexible all-solid-state supercapacitors.

\section{Conflicts of interest}

There are no conflicts to declare.

\section{Acknowledgements}

This work was financially supported by the Technological Innovation Projects in the Hubei Province (2016AAA037).

\section{Notes and references}

1 J.-M. Tarascon and M. Armand, Nature, 2001, 414, 359-367.

2 P. Simon and Y. Gogotsi, Nat. Mater., 2008, 7, 845-854.

3 J. A. Rogers, T. Someya and Y. Huang, Science, 2010, 327, 1603-1607.

4 L. Nyholm, G. Nyström, A. Mihranyan and M. Strømme, Adv. Mater., 2011, 23, 3751-3769.

5 H. Nishide and K. Oyaizu, Science, 2008, 319, 737-738.

6 C. Liu, F. Li, L. P. Ma and H. M. Cheng, Adv. Mater., 2010, 22, E28-E62.

7 X. Yang, J. Zhu, L. Qiu and D. Li, Adv. Mater., 2011, 23, 28332838.

8 B. Xu, S. Yue, Z. Sui, X. Zhang, S. Hou, G. Cao and Y. Yang, Energy Environ. Sci., 2011, 4, 2826-2830.

9 C. X. Guo and C. M. Li, Energy Environ. Sci., 2011, 4, 45044507.

10 G. Xiong, C. Meng, R. G. Reifenberger, P. P. Irazoqui and T. S. Fisher, Electroanalysis, 2014, 26, 30-51.

11 P. Simon and Y. Gogotsi, Acc. Chem. Res., 2012, 46, 10941103.

12 R. Raccichini, A. Varzi, S. Passerini and B. Scrosati, Nat. Mater., 2015, 14, 271-279.

13 C. Liu, Z. Yu, D. Neff, A. Zhamu and B. Z. Jang, Nano Lett., 2010, 10, 4863-4868.

14 Y. Huang, J. Liang and Y. Chen, Small, 2012, 8, 1805-1834.

15 J. Chen, C. Li and G. Shi, J. Phys. Chem. Lett., 2013, 4, 12441253.

16 M. Pumera, Z. Sofer and A. Ambrosi, J. Mater. Chem. A, 2014, 2, 8981-8987.

17 M.-R. Gao, Y.-F. Xu, J. Jiang and S.-H. Yu, Chem. Soc. Rev., 2013, 42, 2986-3017.

18 J. M. Soon and K. P. Loh, Electrochem. Solid-State Lett., 2007, 10, A250-A254.

19 X. Zhang, Z. Sui, B. Xu, S. Yue, Y. Luo, W. Zhan and B. Liu, J. Mater. Chem., 2011, 21, 6494-6497.

20 L. L. Zhang, R. Zhou and X. Zhao, J. Mater. Chem., 2010, 20, 5983-5992.

21 L. L. Zhang, X. Zhao, M. D. Stoller, Y. Zhu, H. Ji, S. Murali, Y. Wu, S. Perales, B. Clevenger and R. S. Ruoff, Nano Lett., 2012, 12, 1806-1812.

22 K. Zhang, L. Mao, L. L. Zhang, H. S. O. Chan, X. S. Zhao and J. Wu, J. Mater. Chem., 2011, 21, 7302-7307.

23 L. Yuan, X.-H. Lu, X. Xiao, T. Zhai, J. Dai, F. Zhang, B. Hu, X. Wang, L. Gong and J. Chen, ACS Nano, 2011, 6, 656661.

24 Q. Wu, Y. Xu, Z. Yao, A. Liu and G. Shi, ACS Nano, 2010, 4, 1963-1970.

25 Y. Sun, Q. Wu and G. Shi, Energy Environ. Sci., 2011, 4, 11131132.

26 M. Pumera, Energy Environ. Sci., 2011, 4, 668-674.

27 F. Liu, S. Song, D. Xue and H. Zhang, Adv. Mater., 2012, 24, 1089-1094.

28 B. G. Choi, J. Hong, W. H. Hong, P. T. Hammond and H. Park, ACS Nano, 2011, 5, 7205-7213.

29 H. Matte, Angew. Chem., Int. Ed., 2010, 49, 4059-4062. 
30 F. Su, C. K. Poh, J. S. Chen, G. Xu, D. Wang, Q. Li, J. Lin and X. W. Lou, Energy Environ. Sci., 2011, 4, 717-724.

31 D. Klemm, F. Kramer, S. Moritz, T. Lindström, M. Ankerfors, D. Gray and A. Dorris, Angew. Chem., Int. Ed., 2011, 50, 54385466.

32 R. J. Moon, A. Martini, J. Nairn, J. Simonsen and J. Youngblood, Chem. Soc. Rev., 2011, 40, 3941-3994.

33 Q. Zheng, Z. Cai and S. Gong, J. Mater. Chem. A, 2014, 2, 3110-3118.

34 H. Sehaqui, Q. Zhou and L. A. Berglund, Compos. Sci. Technol., 2011, 71, 1593-1599.

35 A. Javadi, Q. Zheng, F. Payen, A. Javadi, Y. Altin, Z. Cai, R. Sabo and S. Gong, ACS Appl. Mater. Interfaces, 2013, 5, 5969-5975.

36 W. Chen, H. Yu, Q. Li, Y. Liu and J. Li, Soft Matter, 2011, 7, 10360-10368.

37 A. Isogai, T. Saito and H. Fukuzumi, Nanoscale, 2011, 3, 7185.

38 V. C. Tung, M. J. Allen, Y. Yang and R. B. Kaner, Nat. Nanotechnol., 2009, 4, 25-29.
39 C. Meng, C. Liu, L. Chen, C. Hu and S. Fan, Nano Lett., 2010, 10, 4025-4031.

40 G. Tonoli, E. Teixeira, A. Corrêa, J. Marconcini, L. Caixeta, M. Pereira-da-Silva and L. Mattoso, Carbohydr. Polym., 2012, 89, 80-88.

41 Y. Okita, T. Saito and A. Isogai, Biomacromolecules, 2010, 11, 1696-1700.

42 X.-L. Li and Y.-D. Li, J. Mater. Chem. B, 2004, 108, 1389313900.

43 K. Gao, Z. Shao, J. Li, X. Wang, X. Peng, W. Wang and F. Wang, J. Mater. Chem. A, 2013, 1, 63-67.

44 S. Liu, X. Zhang, H. Shao, J. Xu, F. Chen and Y. Feng, Mater. Lett., 2012, 73, 223-225.

45 A. Di Fabio, A. Giorgi, M. Mastragostino and F. Soavi, J. Electrochem. Soc., 2001, 148, A845-A850.

46 G. Pandey, S. Hashmi and Y. Kumar, J. Electrochem. Soc., 2010, 157, A105-A114.

47 M. Hughes, G. Z. Chen, M. S. Shaffer, D. J. Fray and A. H. Windle, Chem. Mater., 2002, 14, 1610-1613.

48 Y. Wang, Z. Shi, Y. Huang, Y. Ma, C. Wang, M. Chen and Y. Chen, J. Phys. Chem. C, 2009, 113, 13103-13107. 